\title{
Spinocerebellar ataxia type 3 presenting simultaneously with motor neuron disease and cerebellar ataxia
}

Ataxia espinocerebelar tipo 3 apresentando-se simultaneamente com doença do neurônio motor e ataxia cerebelar

Cristina Saade JAQUES', José Luiz PEDROSO', Antônio José da ROCHA², Wladimir Bocca Vieira de Resende PINTO ${ }^{3}$, Acary Souza Bulle OLIVEIRA3 , Orlando Graziani Povoas BARSOTTINI ${ }^{7}$

A 66-year-old man reported an eight-year history of progressive ataxia and one-year of weakness, fasciculation and muscle atrophy (Figure 1). Examination disclosed hypermetric saccades, diffuse fasciculations and absent deep tendon reflexes. Family history was remarkable for autosomal dominant ataxia (Figure 2). MRI showed cerebellar atrophy.

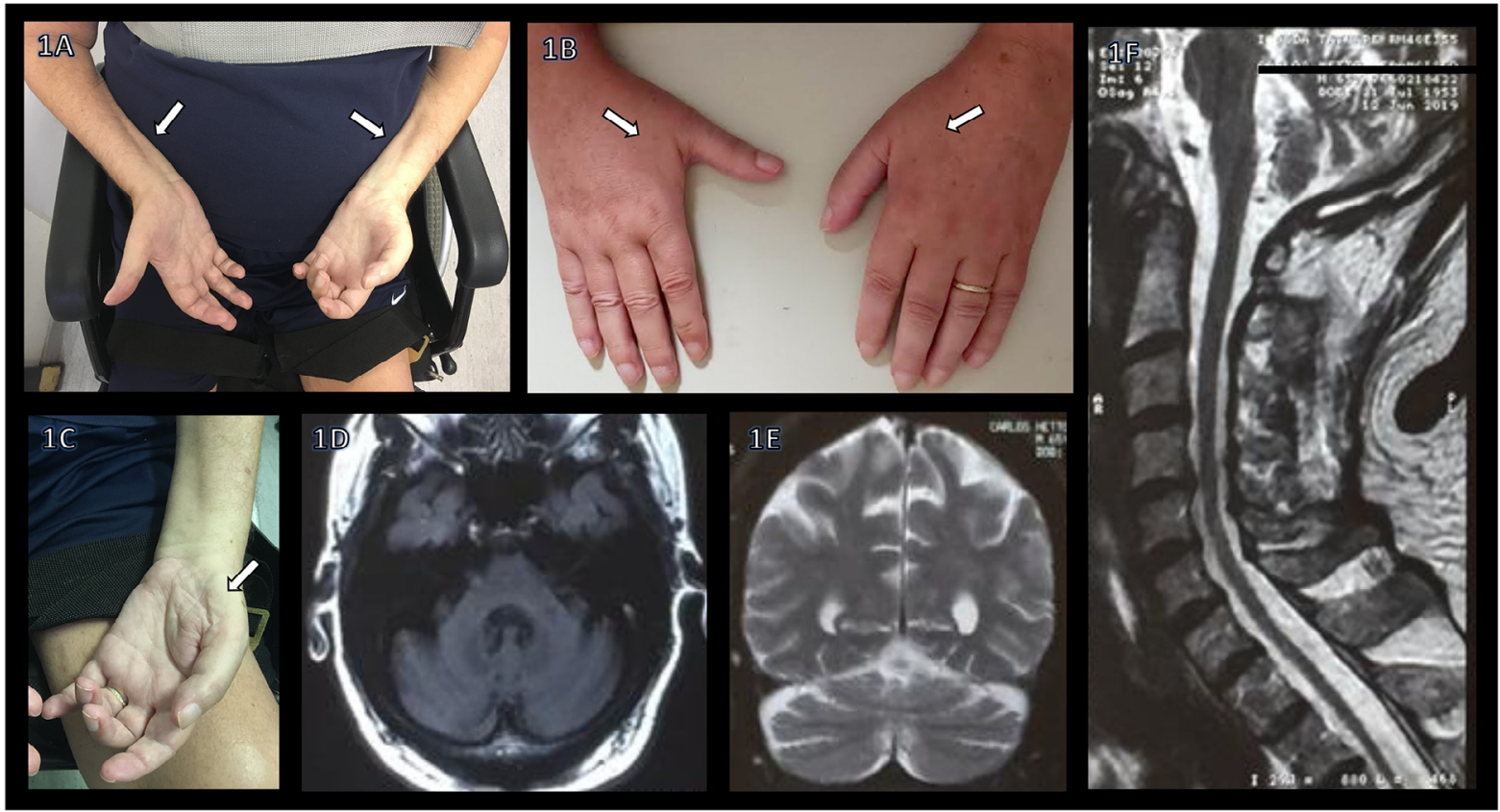

Figure 1. Index patient with spinocerebellar ataxia type 3 and motor neuron disease presenting with forearm flexor muscle atrophy (1A), split hand sign noted as a preferential wasting of first dorsal interossei (1B) and thenar muscles abductor pollicis brevis (1C). Brain MRI showed global diffuse atrophy, more marked on middle and superior cerebellar peduncles, as on cerebellum (1D-E), and cervical spine MRI showed diffuse cervical spinal cord atrophy with no signal changes (1F).

\footnotetext{
'Universidade Federal de São Paulo, Departamento de Neurologia, Unidade de Ataxia, São Paulo, SP, Brazil.

${ }^{2}$ Santa Casa de São Paulo, Faculdade de Ciências Médicas, Divisão de Neurorradiologia, São Paulo, SP, Brazil.

${ }^{3}$ Universidade Federal de São Paulo, Departamento de Neurologia, Unidade Neuromuscular, São Paulo, SP, Brazil.

Cristina Saade Jaques (D) https://orcid.org/0000-0002-2196-3151; José Luiz Pedroso https://orcid.org/0000-0002-1672-8894

Antônio José da Rocha (D) https://orcid.org/0000-0003-2591-9171; Wladimir Bocca Vieira de Resende Pinto (D) https://orcid.org/0000-0002-0150-525X;

Acary Souza Bulle Oliveira (iD https://orcid.org/0000-0002-6986-4937; Orlando G. P. Barsottini (D) https://orcid.org/0000-0002-0107-0831

Correspondence:José Luiz Pedroso; E-mail:zeluizpedroso@yahoo.com.br

Authors' contribution: 1. Case report project:A. Conception, B. Organization, C. Execution; 2. Statistical Analysis: A. Design, B. Execution, C. Review and Critique; 3. Manuscript: A. Writing of the first draft, B. Review and Critique. CSJ:1A, 1B, 1C, 3A, 3B; JLP: 1A, 1B, 3B; AJR: 3B; WBVRP:3B; ASBO: 3B; OGPB: 1A, 1B, 3B.

Ethical statement: Our Ethics Committee has approved this report. Patient provided informed consent for this publication.

Conflict of interest: There is no conflict of interest to declare.

Received on May 02, 2020; Received in its final form on October 09, 2020; Accepted on December 08, 2020.
} 
Genetic testing confirmed SCA3 (66 allelic expansion on ATXN3 gene). EMG disclosed diffuse denervation and confirmed motor neuron disease (MND).

Spinocerebellar ataxias (SCAs) may manifest as MND, particularly SCA2. Although SCA3 usually manifest as peripheral nerve involvement in a neuronopathy pattern and anterior horn degeneration, marked MND is uncommon ${ }^{1,2}$. SCA3 with simultaneous sporadic amyotrophic lateral sclerosis should also be considered and may be related to accumulation of transactivation-responsive DNA-binding protein 43 (TDP-43) in the lower motor neurons ${ }^{3}$.

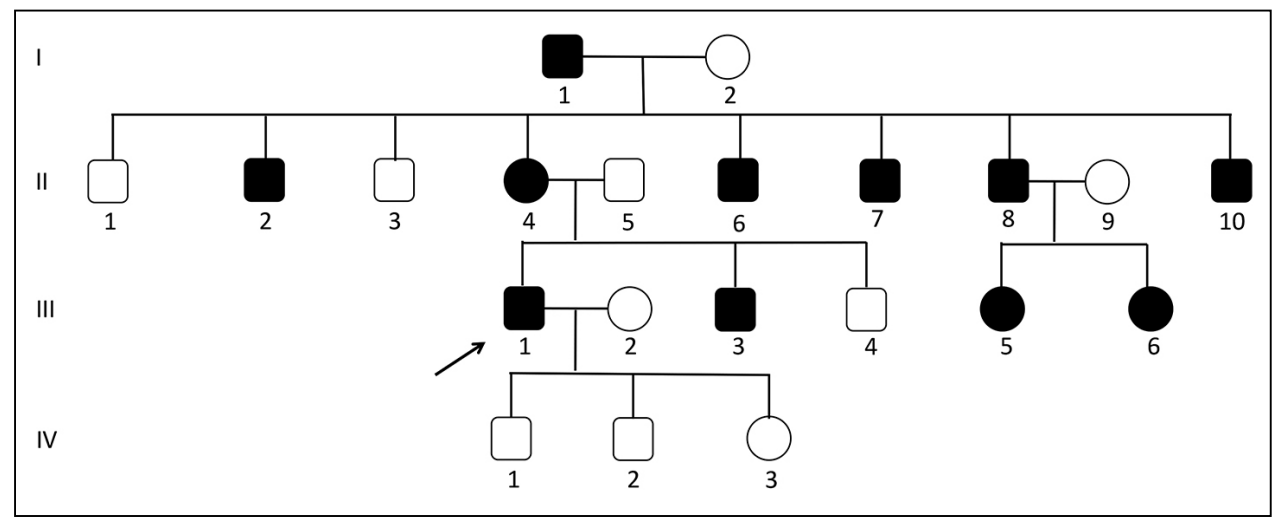

Figure 2. Heredogram. Family with spinocerebellar ataxia type 3 (SCA3).

\section{REFERENCES}

1. Pinto S, De Carvalho M. Machado-Joseph disease presenting as motor neuron disease. Amyotroph Lateral Scler 2008 Jun;9(3):188-91. https://doi.org/10.1080/17482960701702603

2. Moro A, Munhoz RP, Arruda WO, Raskin S, Moscovich M, Teive HA. Spinocerebellar ataxia type 3: subphenotypes in a cohort of brazilian patients. Arq Neuropsiquiatr 2014 Sep;72(9):659-62. https://doi. org/10.1590/0004-282×20140129

3. Toyoshima Y, Takahashi H. TDP-43 pathology in polyglutamine diseases: with reference to amyotrphic lateral sclerosis. Neuropathology. 2014;34(1):77-82. https://doi.org/10.1111/neup.12053 